\title{
Aplications Techniques of Marketing in SME-s in Albania
}

\author{
Azeta Tartaraj \\ Faculty of Business, University "Aleksandër Moisiu" Durres, Albania
}

Doi:10.5901/mjss.2014.v5n23p2755

\begin{abstract}
The SME plays a very important role in the structural organization of production of all type of economies. They have some specific features identified, first of all, in the importance of the entrepreneur and his full involvement in all business activities; which activities are taken in a simple structure of the functional type; a strong presence in industrial markets; and, above all, a frequent absence of precise and formalized strategies and non-use mode of analysis and strategic planning. These characteristics make the SMEs an element in itself that consists on values, behaviors, operating mechanisms and management practices throughout the typical and unique characteristics. If we consider the application of marketing in Albania, the evolution of the discipline is very complex. First, because of the peculiarities and historical evolution of the industrial system, as the SMEs, has facilitated the spread of a feature that requires huge investments, a significant programming skills and organizational resources dedicated and specialized. The objective of this work is to understand what level and state of the art of marketing in small and medium-sized enterprises in Albania, verifying it through some theories of various authors. It then discusses, with several references, the problems of marketing of SMEs with a greater focus on Albanian's SME.
\end{abstract}

Al fine di recuperare vantaggi in un mercato che diventa ogni giorno più competitivo, le imprese dovrebbero riconoscere al marketing il ruolo fondamentale di assicurare la maggiore integrazione possibile tra impresa e mercato, nella concezione estesa a tutte le variabili economiche, istituzionali, sociali, tecnologiche, concernenti la domanda intermedia e finale. A tale scopo, però, il marketing su base intuitiva o empirica dell'imprenditore non è più sufficiente. Infatti, anche chi - dal mondo della professione - sottolinea i successi del marketing delle nostre piccole e medie imprese, ne individua al tempo stesso una debolezza intrinseca, e cioè la scarsità di metodologie codificate di analisi e di azione (analisi della domanda, segmentazione, posizionamento, politiche di marca, fidelizzazione della clientela intermedia e finale) e soprattutto di investimenti in cultura di mercato, quale filosofia che ispiri tutta l'impresa".

Anche Marchini, Cozzi e Ferrero forniscono dei contributi fondamentali per delineare gli aspetti tipici del rapporto tra marketing e PMI.

Ciò, tra l'altro, è in piena consonanza con lo studio di Varaldo, Dalli e Resciniti. I primi puntualizzano, così, sette aspetti cardine di questo rapporto:

1. L'investimento in risorse, competenze e meccanismi di accesso al mercato, ad esempio, in analisi di mercato 0 in meccanismi avanzati di comunicazione con i clienti, è spesso visto come qualcosa di costoso, poco chiaro, apparentemente non redditizio (almeno nel breve termine), non strettamente necessario per il funzionamento dell'impresa e, quindi, dilazionabile;

2. le strutture di marketing sono ridotte al minimo e processi formalizzati di marketing sono quasi del tutto assenti;

3. mancano i presupposti per l'implementazione di un piano di marketing ben strutturato, in quanto molto raramente vengono applicati modelli di segmentazione esplicita e di posizionamento progettuale;

4. si ha un utilizzo molto limitato delle ricerche formali di mercato;

5. la fissazione del prezzo è affidata all'intuizione e alla familiarità con il mercato degli imprenditori;

6. I'attività di vendita si basa essenzialmente sulla rete di rapporti personali dell'imprenditore;

7. si attribuisce molta importanza alla gestione della relazione a scapito di altre leve di natura impersonale, quali la pubblicità e la distribuzione (Marchini, 1997), (Cozzi e Ferrero, 2000).

\section{II marketing processuale e relazionale}

Le caratteristiche riconducibili alla mancanza di una funzione organizzativa dedicata, ad un limitato potere di mercato ed alla ricerca di relazioni strette con i clienti intermedi (che spesso si traducono in una effettiva dipendenza da essi) hanno favorito lo sviluppo nelle PMI di meccanismi di marketing di natura processuale e relazionale. Come sostengono Varaldo, Dalli e Resciniti, "questi hanno sovente permesso di creare valore prescindendo dall'applicazione degli assiomi classici 
del marketing management, ma non essendo sostenuti da investimenti consapevoli hanno finito per rappresentare nel lungo termine essi stessi un fattore di debolezza.

La dimensione processuale del marketing all'albanese s'è sviluppata proprio per la frequente assenza di una funzione organizzativa o di ruoli esplicitamente dedicati ed alla conseguente presenza di processi informali e surrettizi che ne garantiscono l'attuazione, ma al di fuori dei canoni tradizionali. Infatti, lo svolgimento dei processi di marketing avviene in maniera veloce e flessibile sotto il presidio diretto dell'imprenditore, con un approccio decisionale che risulta spesso di tipo trial and error con la negazione di fatto delle logiche di scientific management. Si tratta per lo più di un marketing processuale interno, soprattutto vicino alla produzione, laddove l'integrazione con i distributori è rara (più frequentemente esiste invece con i fornitori). Inoltre, nella pratica la realizzazione dei processi avviene secondo principi opposti a quelli sanciti sul piano teorico, sia per

l'utilizzo degli strumenti di marketing a posteriori, dopo che l'idea del prodotto si è già materializzata e realizzata sul piano manifatturiero, sia per la negazione stessa dei principi fondamentali dell'organizzazione per processi, in particolare l'appiattimento dei livelli di assunzione delle decisioni (è l'imprenditore che progetta e realizza) e l'orientamento al cliente.

La dimensione relazionale è connessa all'assenza di un effettivo potere di mercato (e di marca) che induce le imprese a confrontarsi sui mercati intermedi della distribuzione anziché su quelli finali del consumo e ad affidarsi a relazioni con partner privilegiati che, peraltro, si trasformano spesso in rapporti di dipendenza data la piccola dimensione delle imprese industriali stesse. L'assenza di un brand e l'approccio imprenditoriale più che manageriale, anche in questo caso, consentono l'applicazione di una versione assolutamente limitata dell'approccio relazionale consacrato in letteratura. In essa prevale essenzialmente la dimensione personale delle relazioni rafforzata dai meccanismi di tipo sociale e territoriale del distretto di appartenenza, piuttosto che quella risultante da investimenti volti ad un'effettiva integrazione dei processi.

In prima approssimazione sembra mancare una cultura del valore per il cliente, sia nei settori dei beni di consumo che dei beni industriali. Risulta cioè difficile riscontrare una qualche sensibilità per una formulazione dei problemi gestionali, anche di quelli di marketing, secondo la logica dei bisogni della clientela finale, inquadrati in un paradigma di stampo economico: l'impresa non sembra in grado di progettare value for money per il cliente, partendo dalle sue esigenze e dalla sua disponibilità di risorse e confrontandole con le proprie competenze e il proprio conto economico.

La prima via è rappresentata da progetti di consolidamento delle risorse distrettuali: là dove le piccole imprese non possono più operare da sole, esse possono pervenire a varie forme di cooperazione in grado di realizzare un'offerta fortemente variegata e più strettamente integrata con i network produttivi e logistici internazionali (a monte) e con la distribuzione sia di piccole, sia di grandi dimensioni; in tal modo le imprese minori, senza perdere la propria identità, possono raggiungere le condizioni organizzative e gestionali per riappropriarsi di un qualche controllo del mercato finale di cui i gap dimensionali e di risorse descritti in precedenza le avevano private.

La seconda via concerne l'attuazione di progetti di digitalizzazione: attraverso l'uso di tecnologie digitali e la rete Internet è possibile recuperare l'accesso al mercato anche per realtà industriali di piccole e medie dimensioni, soprattutto per la valorizzazione di risorse locali e tipiche, di natura strettamente tecnica e industriale, ma più in generale legate alla dimensione territoriale e culturale. Gli strumenti di marketing oggi disponibili attraverso la rete sono, neanche troppo paradossalmente, al tempo stesso più articolati e complessi, ma più convenienti. Anche il capitale umano in grado di mettere a disposizione dell'impresa tali risorse comincia ad essere disponibile sul mercato della professione e della formazione universitaria" (Varaldo, Dalli, Resciniti, 2006).

\section{I contributi europei alla teoria del marketing nelle PMI}

Come ben evidenziato da Marchini, Cozzi e Ferrero, relativamente all'applicazione del marketing nelle PMl, si può riconoscere l'esistenza di due principali correnti di pensiero:

1. un'impostazione teorica, riconducibile alla realtà nordamericana, che considera applicabili alle piccole imprese i fondamentali principi del marketing management, con formule e modalità simili a quelle applicate dalle grandi corporation.

2. un'impostazione di stampo europeo, la quale, invece, ritiene necessario rivedere e adattare i metodi e i contenuti del marketing, per renderli più congrui alla realtà delle PMI (Marchini, 1997, Cozzi e Ferrero, 2000).

Delle due correnti, Mattiacci e Ceccotti ritengono sia utile approfondire quella europea perchè, secondo loro, l'impostazione statunitense è un po' troppo rigida e deterministica. A tale scopo, prendono in considerazione il contributo di Carson David J., un autore irlandese che ha elaborato verso la fine degli anni Ottanta un vero e proprio modello tale da essere preso come punto di riferimento per studi successivi di altri autori. 
Essenzialmente, la filosofia di Carson (1985) si fonda su due considerazioni basilari:

I. l'esistenza di una stretta relazione fra il ciclo evolutivo dell'impresa e l'implementazione delle attività di marketing all'interno dell'azienda stessa;

II. la constatazione di un'inadeguata applicazione dei principi generali di marketing management alla realtà delle $\mathrm{PMI}$, le quali necessitano invece di particolari adattamenti rispetto ai modelli più celebri, costruiti e calibrati avendo come termine di riferimento la grande impresa.

II Carson realizza le proprie teorizzazioni partendo dalla realtà delle piccole imprese dell'Irlanda del Nord e cerca di indagare il perché queste organizzazioni non danno al marketing la stessa importanza che le stesse attribuiscono ad altre funzioni aziendali, quali la finanza e la produzione. La prima risposta risiede proprio nelle caratteristiche tipiche delle imprese di dimensioni più piccole e che ovviamente influenzano il modo di affrontare un dato mercato. Su questo terreno, Carson identifica in particolare tre tipi di limitazioni per il marketing delle piccole imprese:

i. Risorse limitate. Le PMI dispongono di poche risorse, sia intermini finanziari, ma anche in relazione alle conoscenze di marketing, di tempo,ecc.

ii. Mancanza di esperti all'interno dell'organizzazione. La funzione marketing viene svolta, molto spesso, dall'imprenditore. Tuttavia, capita di frequente, che questi non disponga delle competenze necessarie per affrontare un ambiente sempre più competitivo e che concentri la sua attenzione esclusivamente sull'attività di vendita e su aspetti prettamente operativi del marketing, sottovalutandone quello strategico (analisi della domanda, posizionamento strategico, e così via). Anche quando l'impresa si sviluppa, passando a un'organizzazione di tipo manageriale, l'acquisizione di eksperti di finanza e di produzione precede normalmente l'ingaggio di uomini di marketing.

iii. Limitato impatto sul mercato. A causa delle limitate risorse finanziarie e della mancanza di esperti di marketing nell'organizzazione, i fattori di marketing sono scarsamente utilizzati. Le piccole imprese hanno spesso un basso numero di clienti e, di conseguenza, un limitato numero di ordini rispetto alle imprese di dimensioni più grandi. Tutto ciò si ripercuote in una bassa notorietà, in una grande difficoltà a emergere e a farsi conoscere all'interno del settore nel quale operano.

Date queste caratteristiche, il Carson si volge poi ad analizzare i cambiamenti che intervengono nelle attività di marketing nel momento in cui l'impresa si sviluppa. In particolare, l'autore individua quattro stadi di evoluzione del marketing delle PMI (Figure 1 e 2).

Carson, infine, circa il quesito inerente l'adattamento o meno di modelli consolidati di marketing delle grandi imprese alle realtà più piccole (filosofia statunitense) esprime il proprio pensiero.

\section{L'evoluzione del marketing nelle PMI secondo Carson (Carson 1985)}

\section{Initial marketing activity (attività iniziali di marketing)}

- I consumatori sono conosciuti e raggiunti direttamente dall'imprenditore

- Principali attenzioni: qualità del prodotto, prezzo, modalità e tempi di consegna

- Uso azzardato e senza coordinamento solo di alcune leve di marketing

2. Reactive selling (vendita reattiva)

- Tutte le attività poste in essere (brochures, lettere promozionali standard) sono del tutto reattive rispetto alle istanze della domanda.

- All'aumentare della domanda e, spesso, della concorrenza nel mercato servito, si avverte la necessità di aumentare le attività di marketing e di ingaggiare un esperto.

- PROBLEMA: le PMI necessitano (per incrementare le vendite e porre in essere un marketing proattivo) di competenze interne, ma le limitate risorse finanziarie e l'esiguo numero di attività da svolgere (almeno inizialmente) non ne consentono l'impiego in condizioni economiche vantaggiose.

- PARADOSSO: un incremento nel livello delle vendite è necessario per giustificare l'ingaggio di un esperto, ma solo con l'intervento di quest'ultimo si potrà avere l'innalzamento sperato delle stesse.

3. Enterpreneurial marketing (marketing imprenditoriale)

- Il paradosso viene risolto dall'imprenditore che inizia a dedicare parte del proprio tempo allo svolgimento delle attività necessarie per incrementare le vendite.

- Le performance delle PMI nel marketing dipenderanno, quindi, dall'attitudine dell'imprenditore in tale ambito.

- PROBLEMA: spesso il proprietario ha una maggiore esperienza e una conoscenza più approfondita degli aspetti produttivi e non del mercato da servire. 
4. Integrated proactive marketing (marketing integrato e proattivo)

- Si tratta di un marketing professionale, portato avanti e applicato da specialisti impiegati all'interno dell'impresa.

- La maggior parte delle PMI non raggiunge l'implementazione di tale struttura di marketing più complessa.

\section{Volume vendite}

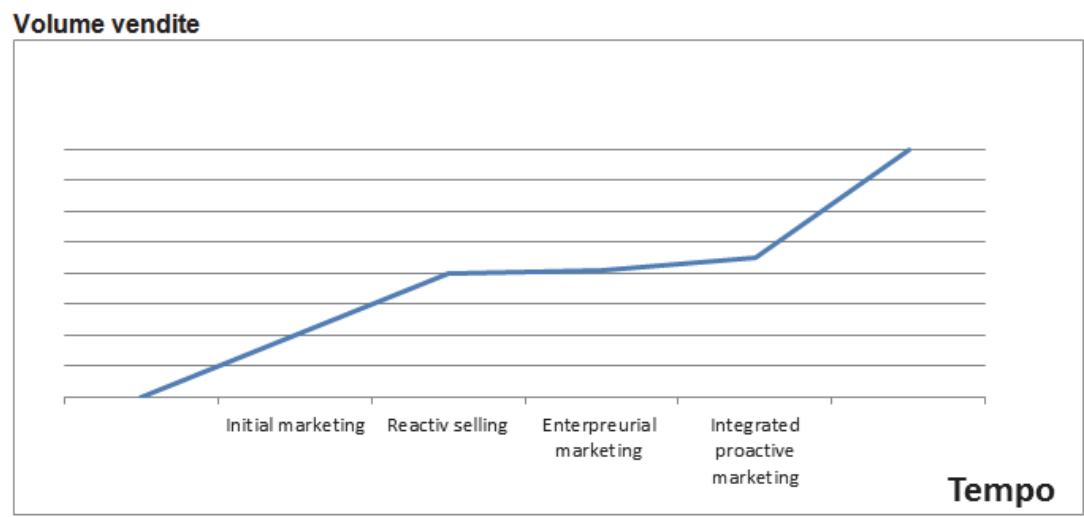

Fig. 1: Gli stadi di sviluppo del marketing nelle PMI (Carson 1985)

Egli afferma che, applicando indistintamente anche alle PMI i principi del marketing management tipici delle imprese di maggiori dimensioni, si rischia di tralasciare le difficoltà e le limitazioni tipiche delle realtà più piccole. I proprietari, infatti, chiamati ad applicare in modo quasi asettico il paradigma delle $4 \mathrm{P}$, si continuerebbero a fare un'idea sbagliata del marketing, visto come un'attività inefficace (date le caratteristiche distinktivë delle imprese di minori dimensioni) e, non riscontrandone l'utilità, continuerebbero a disattenderne i principi e le metodologie.

Il vero imperativo del marketing, invece, risiede nel cambiamento del modo di pensare e di affrontare il mercato che, dunque, non necessariamente deve essere impostato sulla base delle prescrizioni manageriali della grande impresa (Carson, 1985).

\section{Conclusioni}

La probabilità di un proficuo incontro tra le PMl ed il marketing si proponga quale risultato di alcuni elementi:

- il fabbisogno di marketing, ossia l'insorgere, nella proprietà, della percezione che l'approccio al mercato fin lì seguito non sia più idoneo a fronteggiare le mutate condizioni di mercato e delle aziende stesse.

- la considerazione del marketing come voce di costo, a ritorno incerto e non determinabile. Ciò innalza una barriera contro ogni tentativo di operare una svolta nel sistema delle imprese, verso un orientamento scientifico al mercato;

- alcune strutture e processi di gestione del mercato all'interno di una PMI sono legati a prassi consolidate nel tempo, oltretutto cementate spesso dalla natura interpersonale delle relazioni. Questo accento sulla dimensione personale delle relazioni di mercato può porsi come ostacolo al cambiamento;

- il reperimento e il mantenimento di risorse umane qualificate, reso più arduo dalla indeterminatezza delle prospettive di crescita che possono offrirsi all'interno di imprese one-man-band.

Tali considerazioni sono opportune, visto che le prossime strategie imprenditoriale sono quelle di allargamento del mercato ben oltre i confini locali sinora considerati dalle aziende. Ciò, dunque, fa emergere quasi fisiologicamente, all'attenzione del management, tutto l'insieme delle problematiche di marketing, giacchè le piccole e medie imprese si trovano ad affrontare nuove situazioni di scambio, nelle quali non può contare però sulle favorevoli condizioni che ne hanno sostenuto lo sviluppo nel mercato domestico. S'impone, perciò, ad esempio l'adozione di un trade marketing affidato ad un management apposito e che preveda, all'interno di esso, iniziative specifiche inerenti anche la comunicazione aziendale (fiere di settore), laddove invece sul mercato locale ci si affidava alla rete di relazioni sostanzialmente informali costruite dai partner commerciali e dalla forza vendita. I venditori, che rappresentano gli 
ambasciatori dell'impresa e contribuiscono a sviluppare le forme di comunicazione integrata, mediante l'atteggiamento, l'empatia, gli indumenti, la puntualità, la competenza (favorendo la costruzione dell'immagine e della notorietà aziendale) restano, ad ogni modo, le colonne portanti delle aziende.

La notorietà di marca diviene un'arma competitiva importante, laddove si entri in aree dove essa è bassa (o nulla) e si debba fronteggiare una concorrenza popolata di colossi imprenditoriali che investono enormi risorse in comunicazione pubblicitaria e promozionale.

\section{Bibliografia}

Busacca B. (2004). Marketing e creazione di valore. Processi di relazione, customer satisfaction, customer relationship management, processi di misurazione. Teoria e casi, Tools.

Camuffo A., Furlan A., Romano P., Vinelli A. (2004). Crescere e creare valore nei settori maturi: il caso Geox, in Economia \& Management, $n .6$.

Carson D. J. (1985). The evolution of marketing in small firms, Marketing and Small Business (special issue), in European Journal of Marketing, vol. 19, n. 5.

Carson D. J. (1985). The challenge of teaching marketing to small business. Marketing and Small Business (special issue), in European Journal of Marketing, vol. 19, n. 5.

Castaldo S. (2003). Le ricerche per l'analisi delle performance di mercato del prodotto e della marca, in Molteni L. e Troilo G., Ricerche di marketing, McGraw-Hill.

Castaldo S. (2005). Analisi e gestione dei canali distributivi, II Mulino.

Costabile M. (2001). II capitale relazionale, Mc Graw Hill.

Cozzi G. (1986). II marketing nelle piccole e medie imprese, L'economia delle piccole e medie imprese, atti Seminario AIDEA, Clueb.

Cozzi G., Ferrero G. (2000). Marketing: principi, metodi e tendenze evolutive, Giappichelli.

Cugini A., Wilson R. M.S. (2005). Marketing \& Controllo, Egea.

Ferrandina A. (1999). Il marketing strategico per le PMI, FrancoAngeli.

Fornari D. (1999). Rivoluzione commerciale \& Trade Marketing, Egea.

Golfetto F. (2003), Business to business marketing e comunicazione: enfasi sulle competenze del fornitore, in Finanza, marketing e produzione, n.3.

Grandinetti R. (1989). II marketing delle grandi e delle piccole imprese: dalla separazione alla convergenza, in Economia e Politica Industriale, n. 63.

Gronroos C. (2002). Management e marketing dei servizi : un approccio al management dei raporti con la clientela, ISEDI.

Guatri L., Vicari S., Fiocca R. (1999). Marketing, McGraw-Hill.

Guenzi P. (2002). La vendita relazionale. La gestione dei processi commerciali nella prospettiva del Relationship Selling, ETAS.

Guenzi P. (2006). La forza di vendita (cap.12), e Organizzazione e gestione della rete di vendita (cap. 13) in Vicari S., Vendite e Trade Marketing, Egea in collaborazione con il Sole 24 Ore e Repubblica.

Guercini S. (2005). Marketing imprenditoriale, marketing manageriale e conoscenza di mercato del vertice d'impresa, in Mercati e Competitività, n. 1.

Gummesson E. (2002). Total Relationship Marketing, Butterworth Heinemann/Elsevier, Oxford, UK (revised second edition).

Kotler P., Scott W. G. (1993). Marketing management, ISEDI.

Lambin J.J. (2004). Marketing strategico e operativo - Market-driven management, McGraw-Hill.

Lugli G. (1984). Rapporti industria-distribuzione: conflitto o collaborazione?, in Finanza, Marketing e Produzione, n.3.

Lugli G. (1993b). Economia e politiche di marketing delle imprese commerciali, Utet.

Lugli G. (1998). Economia e gestione delle imprese industriali (Trade Marketing), Utet.

Marchini I. (1995). Il governo della piccola impresa. Le basi delle conoscenze, Vol. I, ASPI INSEdit, Genova.

Marchini I. (1997). II difficile rapporto tra piccola impresa e marketing: Io stato delle conoscenze, in Piccola Impresa, n. 1.

Marchini I. (2000). II governo della piccola impresa, Vol. I, Aspi/lns-Edit, Genova.

Mattiacci A., Ceccotti F. (2005). Lo sviluppo del marketing nella Pmi: riflessioni a margine di un'esperienza d'impresa, in Micro\&Macro Marketing, n. 1.

Mauri C. (1996). Come i bambini influenzano gli acquisti, Utet.

Mauri C. (2004). Product \& Brand Management, Egea.

Pastore A., Vernuccio M. (2006).Impresa e comunicazione. Principi e strumenti per il management, Apogeo.

Pencarelli T. (2001). La diffusione di Internet e del commercio elettronico nell'industria turistica nell'esperienza delle imprese alberghiere marchigiane, in Industria e Distribuzione,n. 3.

Ries A., Trout J., (1984). La conquista della posizione vincente, Mc Graw-Hill.

Sciarelli S. (2001). Economia e gestione dell'impresa, Cedam.

Varaldo R., Fornari D. (1998). La evoluzione dei rapporti industria-distribuzione: dalla cooperazione al conflitto, in Sinergie - Rivista di studi e ricerche, n. 46.

Winer R. S. (2002), Marketing management, Apogeo. 